\title{
Fairness Versus Reason in the Ultimatum Game
}

Martin A. Nowak (nowak@ias.edu)

Karen M. Page (page@ias.edu)

Karl Sigmund (karl.sigmund@univie.ac.at)

\section{Approved by}

Ulf Dieckmann (dieckman@iiasa.ac.at)

Project Coordinator, Adaptive Dynamics Network

November 2000 


\section{IIASA STUDIES IN ADAPTIVE DYNAMICS No. 50}

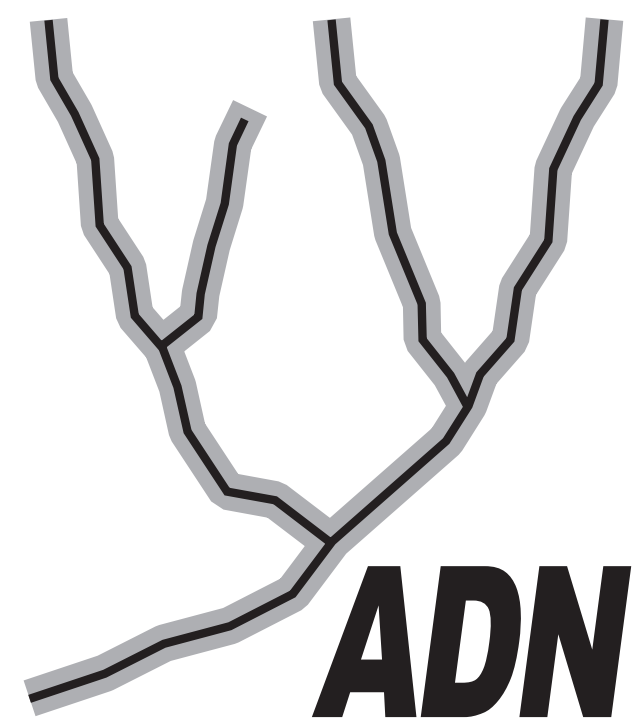

The Adaptive Dynamics Network at IIASA fosters the development of new mathematical and conceptual techniques for understanding the evolution of complex adaptive systems.

Focusing on these long-term implications of adaptive processes in systems of limited growth, the Adaptive Dynamics Network brings together scientists and institutions from around the world with IIASA acting as the central node.

Scientific progress within the network is reported in the IIASA Studies in Adaptive Dynamics series.

\section{THE ADAPTIVE DYNAMICS NETWORK}

The pivotal role of evolutionary theory in life sciences derives from its capability to provide causal explanations for phenomena that are highly improbable in the physicochemical sense. Yet, until recently, many facts in biology could not be accounted for in the light of evolution. Just as physicists for a long time ignored the presence of chaos, these phenomena were basically not perceived by biologists.

Two examples illustrate this assertion. Although Darwin's publication of "The Origin of Species" sparked off the whole evolutionary revolution, oddly enough, the population genetic framework underlying the modern synthesis holds no clues to speciation events. A second illustration is the more recently appreciated issue of jump increases in biological complexity that result from the aggregation of individuals into mutualistic wholes.

These and many more problems possess a common source: the interactions of individuals are bound to change the environments these individuals live in. By closing the feedback loop in the evolutionary explanation, a new mathematical theory of the evolution of complex adaptive systems arises. It is this general theoretical option that lies at the core of the emerging field of adaptive dynamics. In consequence a major promise of adaptive dynamics studies is to elucidate the long-term effects of the interactions between ecological and evolutionary processes.

A commitment to interfacing the theory with empirical applications is necessary both for validation and for management problems. For example, empirical evidence indicates that to control pests and diseases or to achieve sustainable harvesting of renewable resources evolutionary deliberation is already crucial on the time scale of two decades.

The Adaptive Dynamics Network has as its primary objective the development of mathematical tools for the analysis of adaptive systems inside and outside the biological realm. 


\section{IIASA STUDIES IN ADAPTIVE DYNAMICS}

No. 1 Metz JAJ, Geritz SAH, Meszéna G, Jacobs FJA, van Heerwaarden JS:

Adaptive Dynamics: A Geometrical Study of the Consequences of Nearly Faithful Reproduction.

IIASA Working Paper WP-95-099.

In: van Strien SJ, Verduyn Lunel SM (eds.): Stochastic and Spatial Structures of Dynamical Systems, Proceedings of the Royal Dutch Academy of Science (KNAW Verhandelingen), North Holland, Amsterdam, pp. 183-231 (1996).

No. 2 Dieckmann U, Law R:

The Dynamical Theory of Coevolution: A Derivation from Stochastic

Ecological Processes.

IIASA Working Paper WP-96-001.

Journal of Mathematical Biology (1996) 34, 579-612.

No. 3 Dieckmann U, Marrow P, Law R:

Evolutionary Cycling of Predator-Prey Interactions: Population Dynamics and the Red Queen.

Journal of Theoretical Biology (1995) 176, 91-102.

No. 4 Marrow P, Dieckmann U, Law R:

Evolutionary Dynamics of Predator-Prey Systems: An Ecological

Perspective.

IIASA Working Paper WP-96-002.

Journal of Mathematical Biology (1996) 34, 556-578.

No. 5 Law R, Marrow P, Dieckmann U:

On Evolution under Asymmetric Competition.

IIASA Working Paper WP-96-003.

Evolutionary Ecology (1997) 11, 485-501.

No. 6 Metz JAJ, Mylius SD, Diekmann O:

When Does Evolution Optimise? On the Relation between Types of Density

Dependence and Evolutionarily Stable Life History Parameters.

IIASA Working Paper WP-96-004.

No. 7 Ferrière R, Gatto M:

Lyapunov Exponents and the Mathematics of Invasion in Oscillatory or Chaotic Populations.

Theoretical Population Biology (1995) 48, 126-171.

No. 8 Ferrière R, Fox GA:

Chaos and Evolution.

Trends in Ecology and Evolution (1995) 10, 480-485. 
No. 9 Ferrière R, Michod RE:

The Evolution of Cooperation in Spatially Heterogeneous Populations.

IIASA Working Paper WP-96-029.

American Naturalist (1996) 147, 692-717.

No. 10 Van Dooren TJM, Metz JAJ:

Delayed Maturation in Temporally Structured Populations with Non-Equilibrium Dynamics.

IIASA Working Paper WP-96-070.

Journal of Evolutionary Biology (1998) 11, 41-62.

No. 11 Geritz SAH, Metz JAJ, Kisdi É, Meszéna G:

The Dynamics of Adaptation and Evolutionary Branching.

IIASA Working Paper WP-96-077.

Physical Review Letters (1997) 78, 2024-2027.

No. 12 Geritz SAH, Kisdi É, Meszéna G, Metz JAJ:

Evolutionarily Singular Strategies and the Adaptive Growth and Branching of the Evolutionary Tree.

IIASA Working Paper WP-96-114.

Evolutionary Ecology (1998) 12, 35-57.

No. 13 Heino M, Metz JAJ, Kaitala V:

Evolution of Mixed Maturation Strategies in Semelparous Life-Histories: the Crucial Role of Dimensionality of Feedback Environment.

IIASA Working Paper WP-96-126.

Philosophical Transactions of the Royal Society of London Series B (1997) 352, 16471655.

No. 14 Dieckmann U:

Can Adaptive Dynamics Invade?

IIASA Working Paper WP-96-152.

Trends in Ecology and Evolution (1997) 12, 128-131.

No. 15 Meszéna G, Czibula I, Geritz SAH:

Adaptive Dynamics in a Two-Patch Environment: a Simple Model for

Allopatric and Parapatric Speciation.

IIASA Interim Report IR-97-001.

Journal of Biological Systems (1997) 5, 265-284.

No. 16 Heino M, Metz JAJ, Kaitala V:

The Enigma of Frequency-Dependent Selection.

IIASA Interim Report IR-97-061.

Trends in Ecology and Evolution (1998) 13, 367-370. 
No. 17 Heino M:

Management of Evolving Fish Stocks.

IIASA Interim Report IR-97-062.

Canadian Journal of Fisheries and Aquatic Sciences (1998) 55, 1971-1982.

No. 18 Heino M:

Evolution of Mixed Reproductive Strategies in Simple Life-History Models.

IIASA Interim Report IR-97-063.

No. 19 Geritz SAH, van der Meijden E, Metz JAJ:

Evolutionary Dynamics of Seed Size and Seedling Competitive Ability.

IIASA Interim Report IR-97-071.

Theoretical Population Biology (1999) 55, 324-343.

No. 20 Galis F, Metz JAJ:

Why are there so many Cichlid Species? On the Interplay of Speciation and Adaptive Radiation.

IIASA Interim Report IR-97-072.

Trends in Ecology and Evolution (1998) 13, 1-2.

No. 21 Boerlijst MC, Nowak MA, Sigmund K:

Equal Pay for all Prisoners. / The Logic of Contrition.

IIASA Interim Report IR-97-073.

AMS Monthly (1997) 104, 303-307.

Journal of Theoretical Biology (1997) 185, 281-294.

No. 22 Law R, Dieckmann U:

Symbiosis without Mutualism and the Merger of Lineages in Evolution.

IIASA Interim Report IR-97-074.

Proceedings of the Royal Society of London Series B (1998) 265, 1245-1253.

No. 23 Klinkhamer PGL, de Jong TJ, Metz JAJ:

Sex and Size in Cosexual Plants.

IIASA Interim Report IR-97-078.

Trends in Ecology and Evolution (1997) 12, 260-265.

No. 24 Fontana W, Schuster P:

Shaping Space: The Possible and the Attainable in RNA Genotype-Phenotype Mapping.

IIASA Interim Report IR-98-004.

Journal of Theoretical Biology (1998) 194, 491-515.

No. 25 Kisdi É, Geritz SAH:

Adaptive Dynamics in Allele Space: Evolution of Genetic Polymorphism by Small Mutations in a Heterogeneous Environment.

IIASA Interim Report IR-98-038.

Evolution (1999) 53, 993-1008. 
No. 26 Fontana W, Schuster P:

Continuity in Evolution: On the Nature of Transitions.

IIASA Interim Report IR-98-039.

Science (1998) 280, 1451-1455.

No. 27 Nowak MA, Sigmund K:

Evolution of Indirect Reciprocity by Image Scoring. / The Dynamics of Indirect Reciprocity.

IIASA Interim Report IR-98-040.

Nature (1998) 393, 573-577.

Journal of Theoretical Biology (1998) 194, 561-574.

No. 28 Kisdi É:

Evolutionary Branching Under Asymmetric Competition.

IIASA Interim Report IR-98-045.

Journal of Theoretical Biology (1999) 197, 149-162.

No. 29 Berger U:

Best Response Adaptation for Role Games.

IIASA Interim Report IR-98-086.

No. 30 Van Dooren TJM:

The Evolutionary Ecology of Dominance-Recessivity

IIASA Interim Report IR-98-096.

Journal of Theoretical Biology (1999) 198, 519-532.

No. 31 Dieckmann U, O'Hara B, Weisser W:

The Evolutionary Ecology of Dispersal.

IIASA Interim Report IR-98-108.

Trends in Ecology and Evolution (1999) 14, 88-90.

No. 32 Sigmund K:

Complex Adaptive Systems and the Evolution of Reciprocation.

IIASA Interim Report IR-98-100.

Ecosystems (1998) 1, 444-448.

No. 33 Posch M, Pichler A, Sigmund K:

The Efficiency of Adapting Aspiration Levels.

IIASA Interim Report IR-98-103.

Proceedings of the Royal Society of London Series B (1999) 266, 1427-1435.

No. 34 Mathias A, Kisdi É:

Evolutionary Branching and Coexistence of Germination Strategies.

IIASA Interim Report IR-99-014. 
No. 35 Dieckmann U, Doebeli M:

On the Origin of Species by Sympatric Speciation.

IIASA Interim Report IR-99-013.

Nature (1999) 400, 354-357.

No. 36 Metz JAJ, Gyllenberg M:

How Should We Define Fitness in Structured Metapopulation Models? Including an Application to the Calculation of Evolutionarily Stable Dispersal Strategies.

IIASA Interim Report IR-99-019.

Research Report A39 (1999), University of Turku, Institute of Applied Mathematics, Turku, Finland.

No. 37 Gyllenberg M, Metz JAJ:

On Fitness in Structured Metapopulations.

IIASA Interim Report IR-99-037.

Research Report A38 (1999), University of Turku, Institute of Applied Mathematics, Turku, Finland.

No. 38 Meszéna G, Metz JAJ:

Species Diversity and Population Regulation: The Importance of Environmental Feedback Dimensionality.

IIASA Interim Report IR-99-045.

No. 39 Kisdi É, Geritz SAH:

Evolutionary Branching and Sympatric Speciation in Diploid Populations.

IIASA Interim Report IR-99-048.

No. 40 Ylikarjula J, Heino M, Dieckmann U:

Ecology and Adaptation of Stunted Growth in Fish.

IIASA Interim Report IR-99-050.

Evolutionary Ecology (1999) 13, 433-453.

No. 41 Nowak MA, Sigmund K:

Games on Grids.

IIASA Interim Report IR-99-038.

In: Dieckmann U, Law R, Metz JAJ (eds.): The Geometry of Ecological Interactions: Simplifying Spatial Complexity, Cambridge University Press, Cambridge, UK, pp. 135150 (2000).

No. 42 Ferrière R, Michod RE:

Wave Patterns in Spatial Games and the Evolution of Cooperation.

IIASA Interim Report IR-99-041.

In: Dieckmann U, Law R, Metz JAJ (eds.): The Geometry of Ecological Interactions: Simplifying Spatial Complexity, Cambridge University Press, Cambridge, UK, pp. 318332 (2000).

No. 43 Kisdi É, Jacobs FJA, Geritz SAH:

Red Queen Evolution by Cycles of Evolutionary Branching and Extinction. IIASA Interim Report IR-00-030. 
No. 44 Meszéna G, Kisdi É, Dieckmann U, Geritz SAH, Metz JAJ:

Evolutionary Optimisation Models and Matrix Games in the Unified Perspective of Adaptive Dynamics.

IIASA Interim Report IR-00-039.

No. 45 Parvinen K, Dieckmann U, Gyllenberg M, Metz JAJ:

Evolution of Dispersal in Metapopulations with Local Density Dependence and Demographic Stochasticity.

IIASA Interim Report IR-00-035.

No. 46 Doebeli M, Dieckmann, U:

Evolutionary Branching and Sympatric Speciation Caused by Different Types of Ecological Interactions.

IIASA Interim Report IR-00-040.

The American Naturalist (2000) 156, S77-S101.

No. 47 Heino M, Hanski I:

Evolution of Migration Rate in a Spatially Realistic Metapopulation Model.

IIASA Interim Report IR-00-044.

No. 48 Gyllenberg M, Parvinen K, Dieckmann U:

Evolutionary Suicide and Evolution of Dispersal in Structured Metapopulations.

IIASA Interim Report IR-00-056.

No. 49 Van Dooren TJM:

The Evolutionary Dynamics of Direct Phenotypic Overdominance: Emergence Possible, Loss Probable.

IIASA Interim Report IR-00-048.

No. 50 Nowak MA, Page KM, Sigmund K:

Fairness Versus Reason in the Ultimatum Game.

IIASA Interim Report IR-00-057.

Issues of the IIASA Studies in Adaptive Dynamics series can be obtained free of charge. Please contact:

Adaptive Dynamics Network

International Institute for Applied Systems Analysis

Schlossplatz 1

A-2361 Laxenburg

Austria

Telephone +43 2236 807, Telefax +43 2236 71313, E-Mail adn@iiasa.ac.at, Internet http://www.iiasa.ac.at/Research/ADN 


\begin{abstract}
In the Ultimatum Game, two players are offered a chance to win a certain sum of money. All they must do is divide it. The proposer suggests how to split the sum. The responder can accept or reject the deal. If the deal is rejected, neither player gets anything. The rational solution, suggested by game theory, is for the proposer to offer the smallest possible share and for the responder to accept it. If humans play the game, however, the most frequent outcome is a fair share. In this paper, we develop an evolutionary approach to the Ultimatum Game. We show that fairness will evolve if the proposer can obtain some information on what deals the responder has accepted in the past. Hence, the evolution of fairness, similar to the evolution of cooperation, is linked to reputation.
\end{abstract}

\title{
About the Authors
}

\author{
Martin A. Nowak \\ Institute for Advanced Study \\ Einstein Drive \\ Princeton, NJ 08540, USA \\ Karen M. Page \\ Institute for Advanced Study \\ Einstein Drive \\ Princeton, NJ 08540, USA \\ Karl Sigmund \\ Institute of Mathematics \\ University of Vienna \\ Strudlhofgasse 4 \\ A-1090 Vienna, Austria \\ and \\ Adaptive Dynamics Network \\ A-2361 Laxenburg, Austria
}

International Institute for Applied Systems Analysis 


\title{
Fairness Versus Reason in the Ultimatum Game
}

\author{
Martin A. Nowak \\ Karen M. Page \\ Karl Sigmund
}

The Ultimatum Game is quickly catching up with the Prisoner's Dilemma as a prime showpiece of apparently irrational behavior. In the last two decades, it has inspired dozens of theoretical and experimental investigations. The rules of the game are surprisingly simple. Two players have to agree on how to split a sum of money. The proposer makes an offer. If the responder accepts, the deal goes ahead. If the responder rejects, neither player gets anything. In both cases, the game is over. Obviously, rational responders should accept even the smallest positive offer, since the alternative is getting nothing. Proposers, therefore, should be able to claim almost the entire sum. In a large number of human studies, however, conducted with different incentives in different countries, the majority of proposers offer $40-50 \%$ of the total sum, and about half of all responders reject offers below $30 \%^{1-6}$.

The irrational human emphasis on a fair division suggests that players have preferences which do not depend solely on their own payoff, and that responders are ready to punish proposers offering only a small share by rejecting the deal (which costs less to themselves than to the proposers). But how do these preferences come about? One possible explanation is that the players do not grasp that they interact only once. Humans are accustomed to repeated interactions. Repeating the Ultimatum Game is like haggling over a price, and fair splits are more likely ${ }^{6-8}$. Another argument is based on the view that allowing a co-player to get a large share is conceding a relative advantage to a direct rival. This argument holds only for very small groups, however: a simple calculation shows that responders should only reject offers that are less than $1 / N$-th of the total sum, where $N$ is the number of individuals in the group ${ }^{9}$. A third explanation is based on the idea that a substantial proportion of humans maximize a subjective utility function different from the payoff ${ }^{10-12}$.

Here we studied the Ultimatum Game from the perspective of evolutionary game theory ${ }^{13}$. To discuss this model, both analytically and by means of computer simulations, we set the sum which is to be divided equal to 1 , and assume that players are equally likely to be in one of the two roles. Their strategies are given by two parameters $p$ and $q \in[0,1]$. When proposer, the player offers the amount $p$. When responder, the player rejects any offer smaller than $q$. The parameter $q$ can be seen as an aspiration level. It is reasonable to assume that the share kept by the player when proposer, $1-p$, should not be smaller then the aspiration level, $q$. Therefore only strategies with $p+q \leq 1$ were considered $^{14}$.

The expected payoff for a player using strategy $S_{1}=\left(p_{1}, q_{1}\right)$ against a player using $S_{2}=\left(p_{2}, q_{2}\right)$ is given (up to the factor $1 / 2$, which we henceforth omit) by (a) $1-p_{1}+p_{2}$ if $p_{1} \geq q_{2}$ and $p_{2} \geq q_{1}$; (b) $1-p_{1}$ if $p_{1} \geq q_{2}$ and $p_{2}<q_{1}$; (c) $p_{2}$ if $p_{1}<q_{2}$ and $p_{2} \geq q_{1}$; and (d) 0 if $p_{1}<q_{2}$ and $p_{2}<q_{1}$. 
Before studying the full game, with its continuum of strategies, let us first consider a so-called minigame with only two possible offers $h$ and $l$ (high and low), with $0<l<h<1 / 2^{9,15}$. There are four different strategies $(l, l),(h, l),(h, h)$ and $(l, h)$, which we enumerate, in this order, by $G_{1}$ to $G_{4}$. $G_{1}$ is the 'reasonable' strategy of offering little and rejecting nothing (for the cognoscenti: it is the only subgame perfect Nash equilibrium of the minigame $\left.{ }^{16}\right) . G_{2}$ makes a high offer but is willing to accept a low offer. $G_{3}$ is the 'fair' strategy, offering and demanding a high share. For the sake of exposition, we omit $G_{4}$, which gets eliminated anyway. To describe the change in the frequencies $x_{1}, x_{2}$ and $x_{3}$ of the strategies $G_{1}, G_{2}$ and $G_{3}$, respectively, we use the replicator equation. It describes a population dynamics where successful strategies spread, either by cultural imitation or biological reproduction ${ }^{17}$. Under these dynamics, the reasonable strategy $G_{1}$ will eventually reach fixation. Populations that consist only of $G_{1}$ and $G_{3}$ players will converge to pure $G_{1}$ or $G_{3}$ populations depending on the initial frequencies of the two strategies. Mixtures of $G_{1}$ and $G_{2}$ players will always tend to $G_{1}$, but mixtures of $G_{2}$ and $G_{3}$ players are neutrally stable and subject to random drift. Hence, starting with any mixture of $G_{1}$, $G_{2}$ and $G_{3}$ players, evolution will always lead to a population that consists entirely of $G_{1}$ players $^{18}$. Reason dominates fairness.

Let us now introduce the possibility that players can obtain information about previous encounters. In this case, individuals have to be careful about their reputation: if they accept low offers, this may become known, and the next proposer may think twice about making a high offer. Assume, therefore, that the average offer of an $h$-proposer to an $l$ responder is lowered by an amount $a$. Even if this amount is very small - possibly because obtaining information on the co-player is difficult, or because the information may be considered unreliable by $h$-proposers - the effect is drastic ${ }^{19}$. In a mixture of $h$-proposers only, the fair strategy, $G_{3}$ dominates. The whole system is now bistable: depending on the initial condition, either the reasonable strategy $G_{1}$ or the fair strategy $G_{3}$ reaches fixation (Fig. 1). In the extreme case, where $h$-proposers have full information on the responder's type and offer only $l$ when they can get away with it, we observe a reversal of the game: $G_{3}$ reaches fixation while mixtures between $G_{1}$ and $G_{2}$ are neutrally stable. Intuitively, this reversal occurs because it is now the responder who has the initiative: it is up to the proposer to react.

For $0<a<h-l, G_{3}$ risk-dominates ${ }^{20}$ : this implies that whenever one adds stochastic fluctuations to the population (by allowing mutation, for instance, or spatial diffusion) the fair strategy will supersede the reasonable one in the long run (Fig. 1).

Let us now study the evolutionary dynamics on the continuum of all strategies, $S(p, q)$. Consider a population of $N$ players. In every generation, several random pairs are formed. Suppose each player will be proposer on average $r$ times and be responder the same number of times. The payoffs of all individuals are then summed up. For the next generation, individuals leave a number of offspring proportional to their total payoff. Offspring adopt the strategy of their parents, plus or minus some small random value. Thus this system includes selection and mutation. As before, we can interpret these dynamics as denoting biological or cultural reproduction. We observe that the evolutionary dynamics lead to a state where all players adopt strategies that are close to the rational strategy, $S(0,0)$.

Let us now add the possibility that a proposer can sometimes obtain information on what offers have been accepted by the responder in the past. We stress that the same players need not meet twice. We assume that a proposer will offer, whatever is smaller, his own $p$-value or the minimum offer that he knows has been accepted by the responder during previous encounters. In addition, we include a small probability that proposers will make offers that are reduced by a small randomly chosen amount. This effect allows 


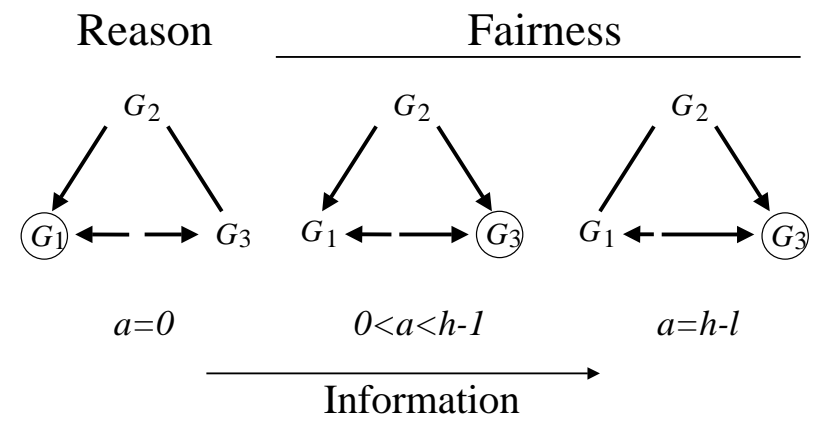

Figure 1: Fairness dominates in the mini-ultimatum game, if proposers have some chance of finding out whether responders might accept a low offer. There are three strategies: the reasonable strategy, $G_{1}(l, l)$, offers and accepts low shares; the fair strategy, $G_{3}(h, h)$, offers and accepts high shares; the strategy $G_{2}(h, l)$ offers high shares but is willing to accept low shares. If there is no information on the responder's type, $a=0$, then the reasonable strategy $G_{1}$ dominates the overall dynamics: $G_{1}$ and $G_{3}$ are bistable, $G_{2}$ and $G_{3}$ are neutral, but $G_{1}$ dominates $G_{2}$. If there is some possibility of obtaining information on the responder's type, then we assume that $h$-proposers will reduce their average offers to $l$-responders by an amount, $a$. For $0<a<h-l$, both $G_{1}$ and $G_{3}$ dominate $G_{2} . G_{1}$ and $G_{3}$ are still bistable, but the fair strategy has the larger basin of attraction; adding noise or spatial affects will favor fairness. In the special limit, $a=h-l$, which can be interpreted as having full information on the responders type, the game is reversed: $G_{1}$ and $G_{2}$ are neutral, while $G_{3}$ dominates $G_{2} ; G_{3}$ is the only strict Nash solution. The figure shows the flow of evolutionary game dynamics ${ }^{17}$ on the edge of the simplex $S_{3}{ }^{18,19}$.

a proposer to test for responders who are willing to accept low offers. Hence, $p$ can be seen as a proposer's maximum offer, while $q$ represents a responder's minimum acceptance level. Each accepted deal is made known to a fraction $w$ of all players. Thus, individuals who accept low offers run the risk of receiving reduced offers in the future. In contrast, the costly act of rejecting a low offer buys the reputation that one accepts only fair offers. Figure 2 shows that this process can readily lead to the evolution of fairness. The average $p$ and $q$ values depend on the number of games per individual, $r$, and the fraction $w$ of individuals who find out about any given interaction. Larger $r$ and $w$ values lead to fairer solutions.

Hence, evolutionary dynamics - in accordance with the predictions of economic game theory - lead to rational solutions in the basic Ultimatum Game. Thus, one need not assume that the players are rational utility-maximizers to predict the prevalence of low offers and low aspiration levels. Whatever the evolutionary mechanism - learning by trial and error, imitation, inheritance - it always promotes the same reasonable outcome: low offers, low demands.

If, however, we include the possibility that individuals can obtain some information on which offers have been accepted by others in previous encounters, the outcome is dramatically different. Under these circumstances, evolutionary dynamics tend to favor strategies that demand and offer a fair share of the prize. This effect, which does not require the same players to interact twice, suffices to keep the aspiration levels high. Accepting low offers damages the individual's reputation within the group and increases the chance of receiving reduced offers in subsequent encounters. Rejecting low offers is costly, but the cost is offset by gaining the reputation of somebody who insists on a fair offer. When reputation is included in the Ultimatum Game, adaptation favors fairness over reason. In this most elementary game, information on the co-player fosters the emergence of strategies that are nonrational, but promote economic exchange. This agrees well with findings on the emergence of cooperation ${ }^{21}$ or of bargaining behavior ${ }^{22}$. Reputation based on commitment and communication plays an essential role in the natural history of economic life ${ }^{23}$. 


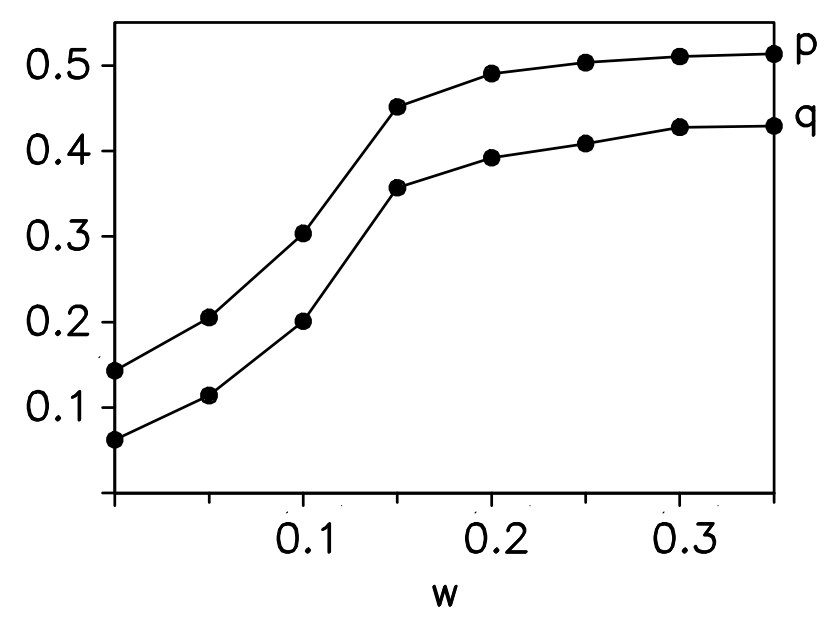

Figure 2: Fairness evolves in computer simulations of the Ultimatum Game, if a sufficiently large fraction, $w$, of players is informed about any one accepted offer. Each player is defined by an $S(p, q)$ strategy with $p+q \leq 1(14)$. In any one interaction, a random pair of players is chosen. The proposer will offer - whatever is smaller - his own $p$ value or the lowest amount that he knows was accepted by the responder during previous interactions. In addition there is a small (0.1) probability that the responder will offer his $p$ value minus some random number between 0 and 0.1 ; this is to test for players who are willing to accept reduced offers. The total population size is $N=100$. Individuals reproduce proportional to their payoff. Offspring adopt their parent's $p$ and $q$ values plus a random number from the interval $(-0.005,+0.005)$. There are on average $r=50$ rounds per player per generation in both roles. Equilibrium $p$ and $q$ values are shown averaged over $10^{5}$ generations. For $w=0$ (no information about previous interactions), the $p$ and $q$ values converge close to the rational solution $S(0,0)$; they are not exactly zero because mutation introduces heterogeneity, and the best response to a heterogeneous population is not $S(0,0)$. For increasing values of $w$, there is convergence close to the fair solution, $S(1 / 2,1 / 2)$, with $q$ being slightly smaller than $p$.

\section{References and Notes}

1. W. Güth, R. Schmittberger, B. Schwarze, J. Econ. Behav. Organ. 3, 376 (1982).

2. $\quad$ R. H. Thaler, J. Econ. Perspect. 2, 195 (1988).

3. W. Güth and R. Tietze, J. Econ. Psychol. 11, 417 (1990).

4. E. Fehr and S. Gächter, Homo Reciprocans and Human Cooperation, Discussion paper, Institute for empirical economic research, University of Zurich (1999).

5. G. E. Bolton and R. Zwick, Game Econ. Behav. 10, 95 (1995).

6. A. E. Roth, in Handbook of Experimental Economics, J. H. Kagel and A. E. Roth, Eds. (Princeton University Press, Princeton, NJ, 1995), 253-348; A. E. Roth, V. Prasknikar, M. Okuno-Fujiwara, S. Zamir, Am. Econ. Rev. 81, 1068 (1991).

7. A. Rubinstein, Econometrica 50, 97 (1982).

8. G. E. Bolton and A. Ockenfels, Am. Econ. Rev. 90, 166 (2000).

9. $\quad$ S. Huck and J. Öchssler, Game Econ. Behav. 28, 13 (1999).

10. G. Kirchsteiger, J. Econ. Behav. Organ. 25, 373 (1994). 
11. J. Bethwaite and P. Tompkinson, J. Econ. Psychol. 17, 259 (1996).

12. D. Kahnemann, J. L. Knetsch, R. Thaler, J. Bus. 59, 5285 (1986); E. Fehr and K.M. Schmidt, Q. J. Econ. 114, 817 (1999).

13. J. Maynard Smith, Evolution and the Theory of Games, (Cambridge University Press, Cambridge; New York, 1982).

14. The condition, $1-p \geq q$, is equivalent to the assumption that the individuals do not regard the role of proposer inferior to the role of responder. Therefore, what they demand for themselves when proposer should not be less than the minimum amount they expect as responders. This condition is relevant when introducing information into the Ultimatum Game. Otherwise, high levels of information can lead to a reversal of the game. If the proposer has perfect knowledge of the responder's $q$ value, then it is in fact the responder who makes the offer. In this case, evolutionary dynamics lead to strategies close to $S(1,1)$, that is proposers have to offer almost the full amount. The condition $1-p \geq q$ avoids this - perhaps unrealistic complication.

15. J. Gale, K. Binmore, L. Samuelson, Game Econ. Behav. 8, 56 (1995).

16. K. G. Binmore, Fun and Games: A Text on Game Theory (D. C. Heath, Lexington, MA, 1992).

17. J. Hofbauer and K. Sigmund, Evolutionary Games and Population Dynamics (Cambridge University Press, Cambridge; New York, 1998).

18. In the minigame we consider the strategies $G_{1}(l, l), G_{2}(h, l), G_{3}(h, h)$ and $G_{4}(l, h)$. The matrix of the expected payoff values is shown in Table 1.

\begin{tabular}{c|cccc} 
& $G_{1}$ & $G_{2}$ & $G_{3}$ & $G_{4}$ \\
\hline$G_{1}$ & 1 & $1-l+h$ & $h$ & $l$ \\
$G_{2}$ & $1-h+l$ & 1 & 1 & $1-h+l$ \\
$G_{3}$ & $1-h$ & 1 & 1 & $1-h$ \\
$G_{4}$ & $1-l$ & $1-l+h$ & $h$ & 0
\end{tabular}

We note first that $G_{4}(l, h)$ is dominated by $G_{1}(l, l)$ so that $x_{4} / x_{1}$ converges to 0 . It follows that all orbits in the interior of the state space $S_{4}$ (the simplex spanned by the unit vectors on the $x_{i}$-axes) converge to the boundary face where $x_{4}=0$. The edge $G_{2} G_{3}$ (high proposers only) consists of fixed points. Those fixed points between $G_{3}$ and $Q(0,[1-h] /[1-l],[h-l] /[1-l], 0)$ are saturated. They cannot be invaded by low proposers and correspond to Nash equilibria. The other points can be invaded by low proposers, $G_{1}$ and $G_{4}$. On the edge $G_{1} G_{2}$, strategy $G_{1}$ dominates. The edge $G_{1} G_{3}$ is bistable, with fixed point $P(1-h, 0, h, 0)$. There is one further fixed point, $R(0,1-h+l, 0, h-l)$ which is stable on the edge $G_{2} G_{4}$ but can be invaded by both missing strategies. The dynamics are simple: orbits can converge either to $G_{1}$, which is the only perfect Nash equilibrium of the game, or else to the segment $Q G_{3}$. But there, neutral drift or recurrent mutations introducing $G_{1}$ or $G_{4}$ will inexorably push the state towards $Q G_{2}$ (it is enough to note that $x_{2} / x_{3}$ grows whenever $x_{1}$ or $x_{4}$ are positive) so that eventually the reasonable strategy $G_{1}$ reaches fixation. 
19. Let us now assume that if a player has a low aspiration level, then this risks to become known to the proposer, in which case an $h$-proposer will, with a certain probability, offer a bit less. Suppose the average offer of an $h$-proposer to an $l$ responder is $h-a$, which is $\geq l$. This implies that the payoff matrix is now given by Table 2 .

\begin{tabular}{c|cccc} 
& $G_{1}$ & $G_{2}$ & $G_{3}$ & $G_{4}$ \\
\hline$G_{1}$ & 1 & $1-l+h-a$ & $h-a$ & $l$ \\
$G_{2}$ & $1-h+l+a$ & 1 & $1-a$ & $1-h+l$ \\
$G_{3}$ & $1-h+a$ & $1+a$ & 1 & $1-h$ \\
$G_{4}$ & $1-l$ & $1-l+h$ & $h$ & 0
\end{tabular}

For $0<a<h-l$, the fair strategy $G_{3}$ dominates $G_{2}$ on the edge $G_{2} G_{3}$. On the edge $G_{1} G_{3}$ the system is bistable: the basin of attraction of $G_{1}$ and $G_{3}$ are separated by the point $P(a)=(1-h+a, 0, h-a, 0)$. The game dynamics have the following properties: (a) The ratio $x_{2} x_{4} / x_{1} x_{3}$ remains constant. (b) There exists a line of fixed points in the interior $S_{4}$, of the form $x_{i}=m_{i}+\mu$ (for $i=1,3$ ) and $x_{i}=m_{i}-\mu($ for $i=2,4)$ where $\mu$ is a real parameter and $\left(m_{1}, m_{2}, m_{3}, m_{4}\right)=$ $(1 / S)(a(1-h), l(1-h), l(h-l-a), a(h-l-a))$ with $S=(l+a)(1-l-a)$. (c) This line intersects all invariant surfaces of the form $x_{2} x_{4}=K x_{1} x_{3}$ (for $K>0$ ) in a fixed point which is a saddle on this surface (the surface is spanned by the edges $G_{1} G_{2} G_{3} G_{4} G_{1}$ ); (d) the corresponding stable manifolds divide the state space $S_{4}$ into two regions, a basin of attraction for $G_{1}$ and a basin of attraction for $G_{3}$; (e) the line intersects either the face $x_{4}=0$ or $x_{2}=0$; the intersection with $x_{4}=0$ is a saddle point $Q(a)$ on this face. There is no other fixed point on this face. If $a$ varies from 0 to $h-l, Q(a)$ describes an arc from the point $Q$ to the point $S(1-l / h, l / h, 0,0)$. For some values of $h$ and $l$, this arc will cross the edge $G_{1} G_{3}$; at such an intersection, $Q(a)=P(a)$. (f) If $a=h-l$ (total knowledge of the co-player, full exploitation of his weakness) we get a phase portrait on the $G_{1} G_{2} G_{3}$-simplex which is exactly the reverse of the phase portrait if $a=0$ : the Nash equilibria are $G_{3}$ and the points on the segment $G_{1} S$. (h) Finally, we note that on the $G_{1} G_{3}$-edge, $P(a)$ separates the basins of attraction: if initially $x_{1}$ is larger than $1-h+a$, then $G_{1}$ reaches fixation. Thus $G_{3}$ has the larger basin of attraction (it is risk-dominant) whenever $h<1 / 2+a$.

20. H. P. Young, Econometrica 61, 57 (1993); M. Kandori, G. J. Mailath, R. Rob, Econometrica 61, 29 (1993); J. Hofbauer, in Game Theory, Experience, Rationality: Foundations of Social Sciences, Economics and Ethics, W. Leinfellner and E. Köhler, Eds. (Kluwer, Dordrecht; Boston, 1998) p. 245.

21. M. A. Nowak and K. Sigmund, Nature 393, 573 (1998); M. A. Nowak and K. Sigmund, J. theor. Biol 194, 561 (1998); A. Lotem, M. A. Fishman, L. Stone, Nature 400, 226 (1999); C. Wedekind and M. Milinsky, Science 288, 850 (2000); A. Zahavi, A. Zahavi, A. Balaban, The Handicap Principle (Oxford Univ. Press, London 1996).

22. T. Ellingsen, Q. J. Econ. 112, 581 (1997); H. P. Young, J. Econ. Theory 59, 145 (1993); A. Banerjee and J. Weibull, in Learning and Rationality in Economics, A. Kirman and M. Salmon, Eds. (B. Blackwell, Oxford; Cambridge, MA, 1995); K. Binmore, A. Shaked, J. Sutton, Am. Econ. Rev. 75, 1178 (1985). 
23. From the huge literature on this topic, we quote two classics: T. C. Schelling, The Strategy of Conflict (Harvard University Press, Cambridge, MA, 1960); R. Frank, Passions within Reason (Norton, New York, 1988).

24. Support from the Leon Levy and Shelby White Initiatives Fund, the Florence Gould Foundation, the J. Seward Johnson, Sr. Charitable Trusts, the Ambrose Monell Foundation and the Alfred P. Sloan Foundation is gratefully acknowledged. 